\title{
Dimensional crossover of heat conduction in low dimensions
}

\author{
L. Yang, ${ }^{1,2,3}$ P. Grassberger, ${ }^{3}$ and B. $\mathrm{Hu}^{2,4}$ \\ ${ }^{1}$ Institute of Modern Physics, Chinese Academy of Science, Lanzhou, China and Department of Physics, \\ Lanzhou University, Lanzhou, China \\ ${ }^{2}$ Department of Physics, and The Beijing-Hong Kong-Singapore Joint Centre for Nonlinear and Complex Systems (Hong Kong), \\ Hong Kong Baptist University, Kowloon Tong, Hong Kong, China \\ ${ }^{3}$ John v. Neumann Institute, Forschungszentrum Jülich, D-52425, Jülich, Germany \\ ${ }^{4}$ Department of Physics, University of Houston, Houston, Texas 77204-5005, USA
}

(Received 12 April 2005; revised manuscript received 16 October 2006; published 8 December 2006)

\begin{abstract}
The dimensional crossover phenomena of heat conduction is studied by a two-dimensional (2D) FermiPasta-Ulam lattice. The 2D divergence law of the thermal conductivity is confirmed by the simulations results. The divergence law of the thermal conductivity will change from the $2 \mathrm{D}$ class to $1 \mathrm{D}$ class as $\delta=N_{y} / N_{x}$ decreases, here $N_{y}$ is the size in transverse direction and $N_{x}$ in longitude direction. The simulation's results suggest that the dimensional crossover happens in $\delta^{*} \rightarrow 0$ as $N_{x} \rightarrow \infty$.
\end{abstract}

DOI: 10.1103/PhysRevE.74.062101

PACS number(s): 05.60.-k, 05.70.Ln, 44.10.+i, 05.45.-a

A long-standing task of nonequilibrium statistic mechanics is the understanding of the underlying dynamical mechanisms, which determine the transport laws. If microscopic interactions between atoms are harmonic, this will lead to the thermal conductivity $\kappa$ diverges by the system size and the Fourier's law will not be obeyed. In 1914, P. Debye had argued that the nonlinearity in the interparticle forces is necessary for Fourier's law to hold. After years of intense studies, there is strong evidence that, in general, the thermal conductivity $\kappa$ would diverge by the system size in onedimensional (1D) isolating systems [1] (here, the "isolating" excludes the systems with on-site potentials). Meanwhile, some 2D isolating systems have been studied, such as the 2D Fermi-Pasta-Ulam (FPU) lattice [2,3], the 2D Toda lattice [5], the 2D Lennard-Jones lattice $[2,4,6]$, and the 2D harmonic disordered lattice [7]. For the 2D FPU lattice, the mode coupling theory (MCT) predicts a logarithmic divergence of the thermal conductivity [4]. However, clear numerical evidence is still lacking and behavior in 2D FPU lattices is unclear [8]. In 2D FPU lattices, the transverse modes will interact with the longitude modes due to the nonlinear interparticle potential, and then the dimensional crossover will happen in the systems. Here, the dimensional crossover not only has attracted attention itself, but also plays a key role for providing clear evidence on the divergence law in 2D systems. The FPU potential has some peculiar features not shared by more realistic systems, however, the basic nonlinearity is included. It is still hopeful to reveal the $2 \mathrm{D}$ divergence law by the concrete case. Furthermore, it is interesting to ask how the divergence law changes from the $2 \mathrm{D}$ class to $1 \mathrm{D}$ class.

In this Brief Report, nonequilibrium molecular dynamics simulations of 2D FPU lattices are performed on extensive system sizes. The Hamiltonian is

$$
H=\sum_{i=1}^{N_{x}} \sum_{j=1}^{N_{y}}\left[\frac{\left|\mathbf{p}_{i j}\right|^{2}}{2 m_{i j}}+V\left(\left|\mathbf{q}_{i+1, j}-\mathbf{q}_{i, j}\right|\right)+V\left(\left|\mathbf{q}_{i, j+1}-\mathbf{q}_{i, j}\right|\right)\right],
$$

where the dimensionless mass $m_{i j}$ is unity, $\mathbf{q}_{i, j}$ the displacement from the equilibrium position $\mathbf{q}_{i, j}^{0}$, and $\mathbf{p}_{i j}$ the corre- sponding momentum vector. The interaction potential takes FPU- $\beta$ potential, $V(x)=\frac{1}{2} x^{2}+\frac{\beta}{4} x^{4}$. Periodic boundary conditions are used in transverse direction. The particles $(1, j), j$ $=1, \ldots, N_{y}$, are in contact with the high temperature heat bath $T_{\text {high }}=22$ and the particles $\left(N_{x}, j\right), j=1, \ldots, N_{y}$, the low temperature heat bath $T_{\text {low }}=12$. The heat baths are implemented as Langevin baths [1]. The definition of the local temperature is $T_{i, j}=\left\langle\left(p_{i, j}^{x}\right)^{2}\right\rangle=\left\langle\left(p_{i, j}^{y}\right)^{2}\right\rangle=\left\langle\left(p_{i, j}^{x}\right)^{2}+\left(p_{i, j}^{y}\right)^{2}\right\rangle / 2$, where $p_{i j}^{x}$ and $p_{i j}^{y}$ are $x$ and $y$ components of the momentum vector $\mathbf{p}_{i, j}$. The $x$ and $y$ components of local heat flux are

$$
\begin{aligned}
& j_{i, j}^{x}=\left[f_{i, j}^{x x}\left(p_{i, j}^{x}+p_{i+1, j}^{x}\right)+f_{i, j}^{y x}\left(p_{i, j}^{y}+p_{i+1, j}^{y}\right)\right] / 4, \\
& j_{i, j}^{y}=\left[f_{i, j}^{x y}\left(p_{i, j}^{x}+p_{i, j+1}^{x}\right)+f_{i, j}^{y y}\left(p_{i, j}^{y}+p_{i, j+1}^{y}\right)\right] / 4,
\end{aligned}
$$

where $\quad f_{i, j}^{x x}=-\frac{\partial V\left(\left|\mathbf{q}_{i+1, j}-\mathbf{q}_{i, j}\right|\right)}{\partial q_{i, j}^{x}}, \quad f_{i, j}^{y x}=-\frac{\partial V\left(\left|\mathbf{q}_{i+1, j}-\mathbf{q}_{i, j}\right|\right)}{\partial q_{i, j}^{y}}, \quad f_{i, j}^{x y}$ $=-\frac{\partial V\left(\left|\mathbf{q}_{i, j+1}-\mathbf{q}_{i, j}\right|\right)}{\partial q_{i, j}^{x}}, f_{i, j}^{y y}=-\frac{\partial q_{i, j}\left(\left|\mathbf{q}_{i, j+1}-\mathbf{q}_{i, j}\right|\right)}{\partial q_{i, j}^{y}}$. Thermal conductivity in longitude direction is obtained by $\kappa=N_{y}^{-1} \sum_{i, j} j_{i, j}^{x} /\left(T_{\text {high }}\right.$ $\left.-T_{\text {low }}\right)$, and $\kappa$ is a sort of "specific conductivity" per "transverse channel." We also can define heat conductivity $\kappa_{\text {trans }}$ along the transverse direction. In all simulations, we checked

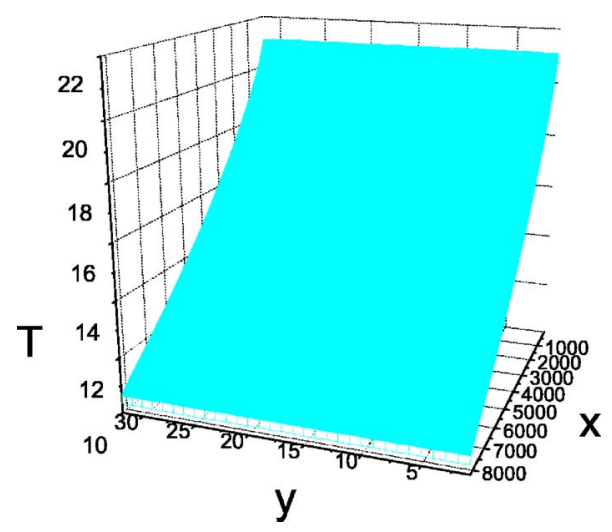

FIG. 1. (Color online) Temperature profile with $N_{y}=32, N_{x}$ $=8192$. In transverse direction $y$, there is no temperature gradient. In longitude direction $x$, the temperature profile is nearly linear. 


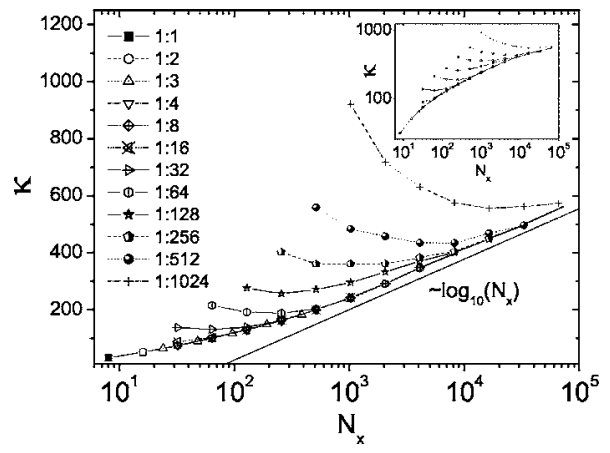

FIG. 2. Plot of $\kappa\left(N_{x}\right)$ with different $\delta=N_{y} / N_{x} . x$ axis is logarithmic scales. The inset shows the same data in the doubly logarithmic scales.

$\kappa_{\text {trans }} / \kappa \lesssim 10^{-5}-10^{-6}$. A leap-frog integrator [9] with a step size 0.025 is used. The time evolution was calculated for time $\simeq 10^{6}-10^{8}$. In all figures of this paper, the statistical errors are always smaller than the data symbols. In the simulations, we take the nonlinear strength $\beta=1$, and the temperature of the heat baths $T>10$. These parameters are chosen to ensure the systems are running in a strong nonlinear regime of FPU potential, then the integrable limit behaviors can be excluded. A typical temperature profile is shown in Fig. 1.

The simulations are performed with the fixed ratio $\delta$ $=N_{y} / N_{x}, \delta$ changes from $1 / 1$ to $1 / 1024$ and $N_{x}=8$ to 65536 , $N_{y}=1$ to 128 . For $N_{x}=65536$, only one datum is obtained at $\delta=1 / 1024 . \kappa\left(N_{x}\right)$ with different $\delta$ are shown in Fig. 2. When $\delta>1 / 8$, the curves overlap. When $\delta<1 / 8$ and $N_{x}$ is large enough, the curves overlap too. When $\delta<1 / 8$ and $N_{x}$ is small, the systems do not bear 2D property due to small $N_{y}$. As $N_{x}<1024$, the overlapped curves show that $\kappa\left(N_{x}\right)$ is linear in $N_{x}=8$ to 64 (the result is the same as Ref. [4]), but $\kappa\left(N_{x}\right)$ in $N_{x}=64$ to 512 shows a clear curving. This indicates a finite size effect. As $N_{x}>1024$, the overlapped curves show a clear linearity by comparing with the black line in a lower position. The black line takes the fitting formula $c_{1}$ $\log _{10} N_{x}+c_{2}$, here $c_{1}$ and $c_{2}$ are arbitrary constants. The loglog plot of the same data, the inset, shows a clear curve. The results support the logarithmic divergence of the thermal conductivity, it is also consistent with the MCT prediction and the long time tail theory.

For a large enough $N_{y}$, the lattice bears the 2D property. As $N_{x}$ becomes larger and larger, the lattice becomes thinner

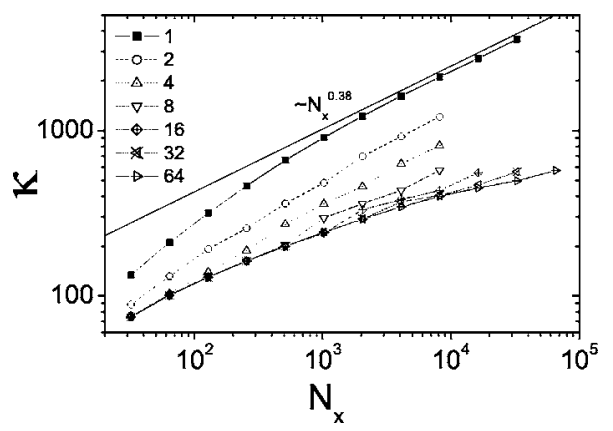

FIG. 3. Log-log plot of $\kappa\left(N_{x}\right)$ with different $N_{y}$ corresponding to the different symbols.

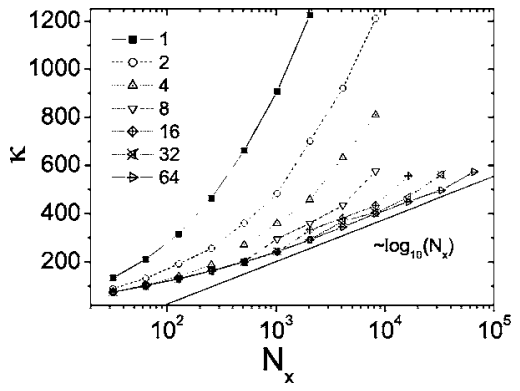

FIG. 4. Plot of $\kappa\left(N_{x}\right)$ with different $N_{y}$ corresponding to the different symbols. $x$ axis is logarithmic scales.

and thinner. The lattice approaches a 1D chain and bears the $1 \mathrm{D}$ property. Then a dimensional crossover from the 2D class to the 1D class will happen. Figure 3 and Fig. 4 show $\kappa\left(N_{x}\right)$ with $N_{y}=1,2,4,8,16,32,64$ for the log-log plot and the lognormal plot, respectively. In Fig. 3, for $N_{y}=1$ (the full square), $\kappa\left(N_{x}\right)$ approaches a line as $N_{x}>1024$ by comparing with the black line in a higher position. The black line takes the fitting formula $c_{1} N_{x}^{0.38}+c_{2}$, here $c_{1}$ and $c_{2}$ are arbitrary constants. Namely, for $N_{y}=1, \kappa\left(N_{x}\right)$ diverges as the power law and belongs to the 1D class. In Fig. 4 , for $N_{y}>8$, the overlapped curves show a clear linearity by comparing with the black line in a lower position. The black line takes a same fitting formula on Fig. 2 including the arbitrary constants. So the overlapped curves show a logarithmic divergence, the 2D class. For fixed $N_{y}$, such as $N_{y}=8$, first $\kappa\left(N_{x}\right)$ belongs to a logarithmic divergence $N_{x}<N_{x}^{*}$, then $\kappa\left(N_{x}\right)$ diverges as the power law as $N_{x}>N_{x}^{*}$. We focus on the aspect ratio $\delta^{*}=N_{y} / N_{x}^{*}$. For $N_{y}=4, N_{x}^{*} \geqslant 200$, namely, $\delta^{*} \leqslant 4 / 200$. For $N_{y}=32, N_{x}^{*} \geq 32000$, then $\delta^{*} \lesssim 32 / 32000$. $N_{x}^{*}$ becomes large and $\delta^{*}$ becomes small as $N_{y}$ increases. Thus we find the dimensional crossover for fixed $N_{y}$. Furthermore, we suggest that the dimensional crossover happens in $\delta^{*} \rightarrow 0$ for $N_{x}$ $\rightarrow \infty$ in the 2D FPU lattice.

The periodic boundary conditions in transverse direction are taken, the simulations are of direct relevance for the thermal conductivity of single-walled tubes. The FPU potential is not a completely realistic description for real tubes. However, as the energy propagates diffusive, it is reasonable that some common features of the thermal conductivity of singlewalled tubes is universal, such as the size dependence and the dimensional crossover. Thus, the size in transverse direction $N_{y}$ can represent the diameter of the tubes. $\kappa\left(N_{y}\right)$ in $N_{x}=32$ to 8192 is shown in Fig. 5. Each $\kappa\left(N_{y}\right)$ will go down

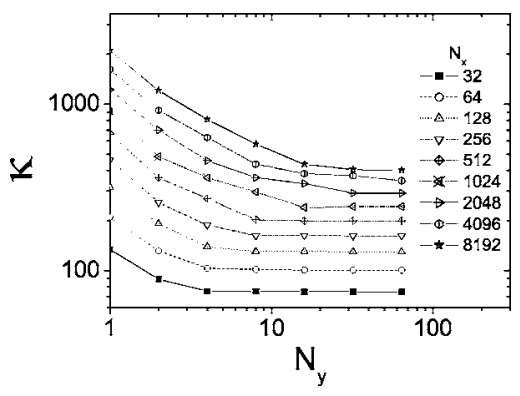

FIG. 5. Log-log plot of $\kappa\left(N_{y}\right)$ with different $N_{x}$. 
as $N_{y}$ increases, then $\kappa\left(N_{y}\right)$ will approach a constant. For the short tube $N_{x}=256, \kappa\left(N_{y}=4\right) / \kappa\left(N_{y}=32\right) \simeq 1.2$. For the long tube $N_{x}=8192, \kappa\left(N_{y}=4\right) / \kappa\left(N_{y}=32\right) \simeq 2.3$. So, the thermal conductivity will be enhanced by the decrease of the diameter.

In summary, we study the size effect and the asymptotic behavior of the thermal conductivity in 2D FPU lattice. For a large $\delta$, the simulations results show that $\kappa$ has a logarithmic divergence, the $2 \mathrm{D}$ class. For a small $\delta, \kappa$ diverges as a power law, the 1D class. We suggest that the dimensional crossover happens in $\delta^{*} \rightarrow 0$ for $N_{x} \rightarrow \infty$. Since the periodic boundary conditions in the transverse direction are used, the simulations actually study the heat conduction of a tube. The thermal conductivity will be enhanced by the decrease of the diameter of the tube, it is a reasonable speculation that the simulations results may indicate the design of cooler for CPU.

We thank Professor Roberto Livi, Antonio Politi, and Walter Nadler for useful discussions. L.Y. acknowledges the support of the 100 Person Project of the Chinese Academy of Sciences. This work was supported in part by grants from the Hong Kong Research Grants Council (RGC) and the Hong Kong Baptist University Faculty Research Grant (FRG).
[1] S. Lepri, R. Livi, and A. Politi, Phys. Rep. 377, 1 (2003).

[2] D. N. Payton, M. Rich, and W. M. Visscher, Phys. Rev. 160, 706 (1967); M. Rich, W. M. Visscher, and D. N. Payton, Phys. Rev. A 4, 1682 (1971).

[3] E. A. Jackson and A. D. Mistriotis, J. Phys.: Condens. Matter 1, 1223 (1989).

[4] A. Lippi and R. Livi, J. Stat. Phys. 100, 1147 (2000).

[5] N. Nishiguchi, Y. Kawada, and T. Sakuma, J. Phys.: Condens. Matter 4, 10227 (1992); J. Michalski, Phys. Rev. B 45, 7054 (1992).
[6] R. D. Mountain and R. A. MacDonald, Phys. Rev. B 28, 3022 (1983).

[7] L. Yang, Phys. Rev. Lett. 88, 094301 (2002); B. Hu, B. Jin, L. Wang, and H. Yang, ibid. 90, 119401 (2003); L. Yang, ibid. 90, 119402 (2003); L. Lee and A. Dhar, ibid. 95, 094302 (2005).

[8] S. Lepri, R. Livi, and A. Politi, Chaos 15, 15118 (2005).

[9] D. J. Evans and G. P. Morriss, Statistical Mechanics of Nonequilibrium Liquids (Academic, San Diego, 1990). 